
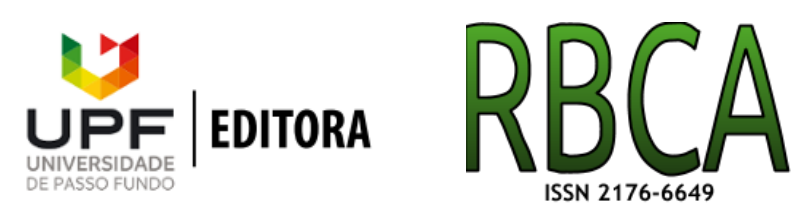

Revista Brasileira de Computação Aplicada, Novembro, 2021

DOI: 10.5335/rbca.v13i3.11093

Vol. 13, № 3, pp. 22-31

Homepage: seer.upf.br/index.php/rbca/index

ARTIGO ORIGINAL

\title{
O professor como coordenador em um ambiente móvel colaborativo de aprendizagem
}

\section{The teacher as coordinator in a mobile collaborative learning environment}

\author{
Anderson Prante ${ }^{(\mathbb{D}, 1}$ and Carla Diacui Medeiros Berkenbrock ${ }^{(\mathbb{D}, 1}$ \\ ${ }^{1}$ Universidade do Estado de Santa Catarina - UDESC \\ *andersonprante@gmail.com; carla.berkenbrock@udesc.br;...
}

Recebido: 26/05/2020. Revisado: 16/06/2021. Aceito: 11/10/2021.

\section{Resumo}

Em ferramentas de aprendizagem colaborativa apoiadas por dispositivos móveis, trocas de mensagens são realizadas pelos usuários para apoiar a construção coletiva de conhecimento pelos membros de um grupo. $O$ registro das atividades ocorridas no grupo fornecem indícios de como ocorre a evolução da aprendizagem. Mecanismos de coordenação em conjunto com técnicas de learning analytics podem ser utilizados em ambientes de ensino, para apoiar o professor na coordenação, possibilitando-o adaptar suas propostas pedagógicas. Essa pesquisa visa identificar os mecanismos de coordenação para ambientes colaborativos de ensino-aprendizagem móvel, bem como aplicá-los à modelagem de um sistema (EZClass) com ênfase na coordenação do professor em um ambiente virtual de aprendizagem. Os mecanismos são representados por meio de requisitos funcionais e são utilizados na definição do sistema EZClass. O EZClass surge com a proposta de auxiliar o professor na coordenação de atividades em um ambiente virtual de aprendizagem, de modo que o professor possa tomar decisões com base nos indicadores apresentados, permitindo-o adaptar sua proposta pedagógica para melhor atender a demanda dos alunos.

Palavras-Chave: Coordenação; Learning Analytics; Sistemas Colaborativos.

\begin{abstract}
In collaborative learning tools supported by mobile devices, users exchange messages in order to support the collective construction of knowledge by the members of a group. Activity entries in the group provide evidence of how the learning progress occurs. Coordination mechanisms together with learning analytic techniques can be used in teaching environments, in order to support the teacher in the coordination, allowing him/her to adapt the pedagogical proposals. This paper aims to identify the coordination mechanisms for mobile teaching-learning collaborative environments, as well as to apply them to model a system (EZClass) with emphasis on teacher coordination in a virtual learning environment. The mechanisms are represented by functional requirements and they are used in the definition of the EZClass system. The EZClass was designed to assist the teacher in coordinating activities on a virtual learning environment, so that the teacher can make decisions based on the presented indicators, allowing to adapt the pedagogical proposal to better supply the students' demands.
\end{abstract}

Keywords: Collaborative Systems; Coordination; Learning Analytics. 


\section{Introdução}

Sistemas colaborativos são sistemas computacionais caracterizados por auxiliar um grupo de pessoas que trabalham em conjunto para realizar uma tarefa ou objetivo comum, por meio de um ambiente de interface compartilhada, procurando manter um contexto comum entre os participantes (Ellis et al., 1991). Os sistemas colaborativos são sistemas baseados em tecnologias de computação e telecomunicações que auxiliam grupos de usuários a exercer uma atividade. Enquanto o termo Sistemas Colaborativos refere-se à tecnologia, a área denominada CSCW (Computer Supported Cooperative Work), em português, Trabalho Cooperativo Auxiliado por Computador, refere-se a um grupo de pessoas que trabalham de forma colaborativa no mesmo campo de pesquisa (Grudin, 2001). Greenberg (1989) considera que CSCW pode ser caracterizado como uma disciplina científica que guia o projeto e o desenvolvimento de sistemas colaborativos de forma detalhada e apropriada.

Do mesmo modo que a área de CSCW, a área da Aprendizagem Cooperativa Apoiada por Computador ou Computer Supported Cooperative Learning (CSCL) tem como estratégia analisar de que forma o uso da tecnologia pode apoiar no processo colaborativo, porém, nesse caso, trata-se especificamente dos processos de aprendizagem (Felder and Brent, 2007).

Ambas as áreas CSCW e CSCL utilizam-se de sistemas computacionais para permitir que trabalhos colaborativos sejam realizados e, segundo Pimentel et al. (2006), o sucesso da colaboração em sistemas colaborativos depende de três fatores: a comunicação, a coordenação e a cooperação dos envolvidos. Esses fatores são representados no modelo 3C de colaboração.

O modelo de colaboração 3C surgiu por volta da década de 1990 (Ellis et al., 1991) e foi adaptado por Fuks et al. (2003). O modelo 3C é utilizado no desenvolvimento de ferramentas colaborativas, sendo que: a comunicação compreende a troca de mensagens e a negociação de compromissos; a cooperação envolve o trabalho em conjunto dos elementos da equipe no espaço compartilhado; e na coordenação as pessoas, as tarefas e os recursos são geridos para lidar com conflitos de interesse apoiando a comunicação e a cooperação.

A aprendizagem colaborativa tem sido defendida e praticada por muitos professores, surgindo dela vários benefıcios como a competência para resolver problemas de grande porte, a partir das contribuições individuais de cada participante (Pasini, 2020). Assim como o computador de mesa, os dispositivos móveis podem ser considerados um recurso para a aprendizagem colaborativa, utilizados para que os alunos colaborem uns com os outros nas atividades em grupo (Varella et al., 2002). Torres et al. (2004) completam que a aprendizagem colaborativa é também uma estratégia de ensino que encoraja a participação dos estudantes no processo de aprendizagem e que faz da aprendizagem um processo ativo e efetivo.

Algumas ferramentas têm sido propostas para fomentar o aprendizado colaborativo por meio de dispositivos móveis. Trabalhos como de Correa and Shinaigger (2020), dos Santos Fernandes and Brasileiro (2020), Nascimento and Filho (2016), Barbour et al. (2014), Ting (2013), Ros- chelle et al. (2010) têm verificado o potencial da utilização de dispositivos móveis na educação, bem como os benef1cios de incorporá-los em sala de aula para promover a aprendizagem colaborativa e participação ativa dos alunos. O crescente avanço de tecnologias móveis propicia o surgimento de aplicações móveis. Por exemplo, na área da aprendizagem móvel, é possível, por meio da mobilidade do usuário e dos dispositivos, prover recursos de aprendizagem em vários contextos, envolvendo diferentes espaços, tarefas e modos de interação.

No desenvolvimento de tecnologias de informação e comunicação que apoiem a coordenação, se faz necessário que os desenvolvedores entendam o fluxo de trabalho e o fluxo das informações, para poder identificar as potenciais áreas de ruptura. A coordenação envolve a compreensão e elaboração de planos de ações, para a mobilização de equipes, avaliação de riscos, priorização de objetivos, decisões rápidas e coordenação de recursos (Arnaut et al., 2016).

Nesse contexto o uso de Learning Analytics pode valerse como um instrumento para auxiliar o professor/coordenador do ambiente online, permitindo-o acompanhar as atividades e coordenar as tarefas. Learning Analytics surgiu da necessidade de se analisar os dados gerados a partir das interações entre alunos nos ambientes virtuais de aprendizagem (AVA) com objetivo de contribuir para a melhoria no processo de ensino aprendizagem (Junior and Oliveira, 2016). Contudo, um dos problemas na utilização de learning analytics está relacionado com a falta de clareza a respeito do que deve ser mensurado para a efetiva compreensão da aprendizagem (Duval, 2011).

Com base nos registros das interações dos estudantes no ambiente virtual é possível extrair medidas quantitativas que podem ser analisadas e que possibilitam compreender a efetividade das estratégias pedagógicas utilizadas (Silva et al., 2016). Como observado, para possibilitar a análise de desempenho e engajamento nas atividades, os sistemas apresentam gráficos que sumarizam informações referente ao número de acessos realizados à áreas específicas no sistema (Ramos et al., 2014).

Machado et al. (2018) desenvolveram uma ferramenta chamada de CLinClass, utilizada para apoiar a aprendizagem colaborativa a partir de dispositivos móveis. Para o desenvolvimento da ferramenta, foram definidos os requisitos de aprendizagem e colaboração necessários para que a ferramenta pudesse auxiliar no processo do aprendizado colaborativo. Focada exclusivamente no trabalho cooperativo dos alunos, a ferramenta não fornece recursos para que o professor possa acompanhar o andamento das atividades ou ainda, visualizar os dados de atividades que já ocorreram.

Como forma de ampliar os recursos providos pela ferramenta CLinClass, descrevemos os mecanismos de coordenação que, em conjunto com as técnicas de learning analytics, foram utilizadas no levantamento de métricas orientadas a auxiliar na análise do processo de aprendizagem. Adicionalmente, também descrevemos de que modo essas métricas podem ser apresentadas em uma ferramenta digital. Os dados educacionais obtidos são apresentados por meio de representação gráficas e relatórios, oferecendo ao professor a possibilidade de realizar alterações em sua proposta pedagógica. 


\section{Trabalhos Correlatos}

Os trabalhos correlatos apresentam técnicas, recursos ou características que tem como objetivo fornecer formas de extração de dados bem como a apresentação de informações aos professores e usuários dos ambientes de aprendizagem.

Silva et al. (2016) apresentam uma abordagem de integração do framework Shiny com o sistema de gestão de aprendizagem (LMS) Moodle para construção de learning analytics. Para a realização dos estudos foi desenvolvida uma ferramenta web que coleta as informações do LMS. O framework Shiny foi escolhido, segundo os autores, pois facilita o desenvolvimento de aplicações web, o que possibilita a construção de interfaces interativas, além de ser multiplataforma. Para a execução dos testes, foi utilizada a instância com dados exemplares da plataforma Moodle, disponível publicamente para experimentação e demonstração. As informações apresentadas pela solução proposta se referem ao número de acessos as áreas do sistema, aos fóruns e aos números de mensagens criadas nos fóruns. Essas informações são apresentadas por meio de gráficos do tipo bolha e tabelas que possuem a capacidade de filtrar um intervalo de dados com base no período das informações.

Stephens-Martinez et al. (2014) realizaram um estudo a respeito de quais informações são necessárias para que um instrutor de cursos online possa entender sua turma. A pesquisa foi realizada com 92 instrutores de ambientes MOOC (Massive Open Online Course), a qual consistiu-se de cinco etapas, sendo algumas delas a realização de um levantamento sobre os instrutores, o desenvolvimento de um protótipo com cinco gráficos diferentes de informações para o monitoramento do MOOC e também a realização de perguntas a respeito da eficiência dos gráficos. O protótipo "Metrics Tab" teve como objetivo fornecer aos instrutores um painel com informações relevantes do curso. Contudo, os autores relatam que saber a quantidade de postagens que um aluno realiza não é tão relevante quanto saber o que é discutido nas postagens, sendo necessário que o professor acompanhe o debate.

Mazza and Milani (2004) apresentam a ferramenta GISMO, um sistema de monitoramento e rastreamento de estudantes que usa os dados de rastreamento dos alunos do Moodle. O sistema transforma os dados em uma forma conveniente para o processamento e gera representações gráficas que podem ser exploradas e manipuladas por instrutores de cursos para examinar comportamentos sociais, cognitivos e aspectos comportamentais dos estudantes à distância. Como resultado, os autores relatam que foi realizada uma avaliação empírica das representações gráficas. Uma amostra de instrutores revelou que as representações gráficas produzidas com o GISMO podem ajudar os instrutores a identificar indivíduos que precisam de atenção especial, descobrir padrões/tendências em acessos e discussões e refletir sobre suas práticas de ensino.

Filvà et al. (2014) utilizaram o google analytics como ferramenta para realizar o rastreamento temporal dos estudantes no ambiente virtual Moodle. Para a coleta das métricas foi desenvolvido uma extensão para o navegador Google Chrome. Todos os participantes precisaram instalar essa extensão no navegador para que as métricas pudessem ser coletadas. Além da instalação da extensão, se fez necessária a adição de um trecho de código no código fonte do Moodle para que o google analytics pudesse funcionar. A ferramenta do google analytics permitiu coletar métricas como: tempo gasto por estudante em um atividade, tempo total gasto por estudante durante as atividades, data e hora de acessos dos estudantes. Segundo os autores, a análise dos gráficos gerados com base no tempo gasto no ambiente virtual ajudam a detectar possíveis desistências ou desengajamentos dos alunos.

da Silva Marques et al. (2021) realizaram uma análise acerca de trabalhos com resultados sobre o uso de aplicativos de mensagem instantânea para promoção da aprendizagem. Os trabalhos selecionados para análise descrevem as vantagens e desvantagens identificadas de acordo com cada área de estudo, como física (1); língua estrangeira (3); e medicina (1). Entre as desvantagens citadas estão a ausência de gestão de respostas e comentários, falta de estímulo na autoaprendizagem e incentivo a interações; e entre as vantagens estão o rápido compartilhamento de mensagens, áudios, vídeos e documentos, a criação de grupos. Ao concluir os autores descrevem que os aplicativos de mensagens instantâneas podem ser utilizados como uma ferramenta para a disseminação de informações, contribuição na aprendizagem dos alunos e melhora na permanência dos participantes nos estudos por meio da manutenção do contato. Não obstante, concluem que é importante que novos estudos sejam realizados em diferentes contextos de aprendizagem.

Chorfi et al. (2020) apresentam uma solução concebida para aprendizagem colaborativa com viés de melhorar as habilidades de resolução de problemas em linguagens de programação. A solução implementada oferece 4 modos de colaboração, a saber: (i) responsabilidade individual onde os indivíduos são responsáveis por uma parte do problema e somente um é capaz de modificar o problema na sua totalidade; (ii) trabalhos alternativos - cada indivíduo realiza a sua implementação para o problema proposto e elas são incorporadas em uma solução única ao final do trabalho; (iii) troca de tarefas dinâmicas - ao longo da sessão de trabalho os indivíduos podem trocar as partes do problema a qualquer momento; e (iv) responsabilidade coletiva - neste modo os alunos desenvolvem juntos uma solução em comum, engajando-os na tomada de decisão para a resolução dos problemas apresentados. Os autores relatam que é necessário um estudo mais aprofundado para comprovação da eficácia na aprendizagem, porém, que em sessões de aprendizagem reais onde envolve a resolução de programação a ferramenta PBPCLG teve um efeito positivo comportamental na maneira em que tutores e alunos à utilizaram.

\section{Ferramenta Colaborativa de Aprendizagem}

O CLinClass é um aplicativo em JavaScript para dispositivos móveis que tem por objetivo facilitar e apoiar a construção de um ambiente de aprendizagem. $O$ aplicativo tem como funcionalidades o acompanhamento do andamento e quantidade de acertos das atividades de grupo, a visualização dos resultados dos alunos, do ranqueamento das questões e do status geral das atividades e a possibilidade 
de correção manual das questões.

o CLinClass implementa uma abordagem denominada ciclo de sessão colaborativa, que tem como objetivo, possibilitar práticas educacionais diferenciadas daquelas que tradicionalmente o professor expõe o conteúdo de um assunto e em seguida aplica uma lista de tarefas de modo a fixar o conteúdo apresentado. Machado et al. (2018) descrevem que, essa abordagem fundamenta-se no conceito de sala de aula invertida e na prática da aprendizagem colaborativa, sendo o momento individual e em grupo complementares. O que permite ao professor adaptar sua aplicação conforme o plano de trabalho e o nível de compreensão dos alunos. O ciclo de sessão colaborativo reúne duas estratégias de trabalho: na primeira, os alunos trabalham individualmente e depois em grupo; e na segunda estratégia, os alunos trabalham diretamente em grupo, conforme apresentado na Fig. 1.

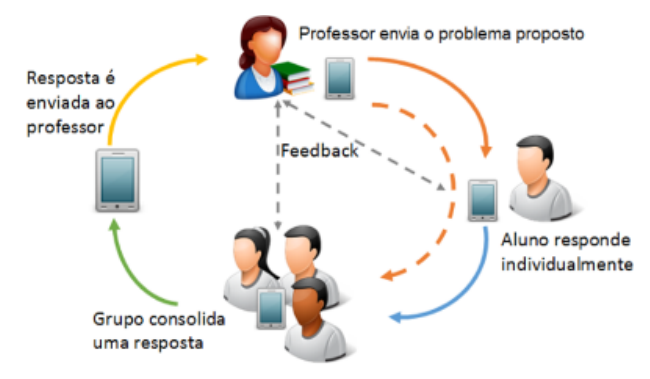

Figura 1: Ciclo de sessão colaborativo de Machado et al. (2018).

Com a aplicação do ciclo de sessão, e, após a realização individual de uma proposta de trabalho, os alunos formam grupos para compartilhar suas conclusões, e juntos, chegar à um consenso. Uma vez encontrada a resposta final, ela é encaminhada para o professor por meio do aplicativo CLinClass.

O CLinClass permite que um professor elabore atividades com uma abordagem definida como ciclo de sessão colaborativa. Nesta abordagem o docente pode utilizar a estratégia do trabalho individual seguido de trabalho em grupo, ou a estratégia dos alunos trabalharem diretamente em grupo, com o propósito de, fomentar a construção coletiva do conhecimento.

O CLinClass detêm-se ao processo de aprendizagem, onde o foco é o aluno. Verificado a insuficiência de recursos para que o professor acompanhe as atividades realizadas no ambiente virtual, a Seção 4 apresenta uma ferramenta denominada EZClass, desenvolvida neste trabalho com a finalidade de proporcionar ao docente recursos para que ele seja capaz de visualizar o andamento das atividades colaborativas realizadas no CLinClass.

\section{EzClass}

O EZClass é uma ferramenta web que tem por finalidade integrar-se a base de dados do CLinClass extraindo informações e as apresentando de modo que possibilite ao professor identificar o nível de engajamento dos alunos nas atividades bem como o nível de desempenho de alunos.

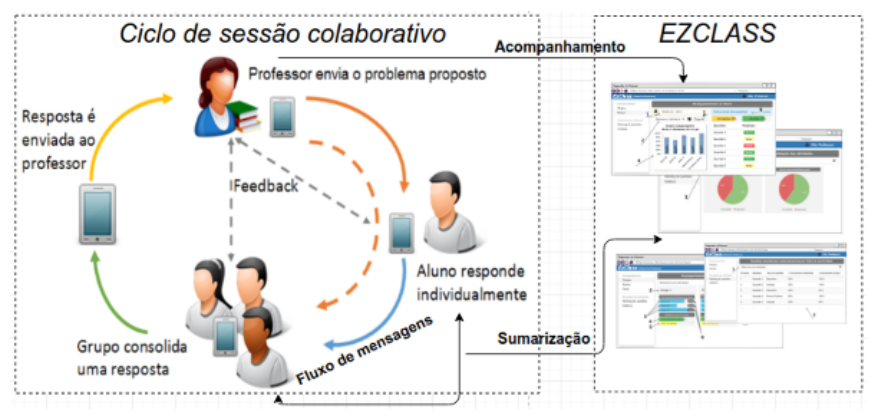

Figura 2: Ciclo de sessão colaborativo adaptado.

O ciclo de sessão colaborativo descrito inicialmente na Seção 3, representado na Fig. 2, foi adaptado para representar a forma no qual o EZClass pode monitorar as ações dos usuários. O EZClass tem como característica fornecer gráficos e tabelas que podem ser utilizados pelo professor a qualquer momento para visualizar o resultado de uma atividade após ser finalizada ou ainda acompanhar uma atividade em andamento. Dentre os tipos de gráficos utilizados estão, o gráfico de barras de progresso para apresentar a quantidade de questões já respondidas por aluno, o progresso geral do grupo, um quantificador de acertos das questões já respondidas e gráficos do tipo pizza para comparação de desempenho entre as atividades realizadas em grupo e individual. O EZClass proporciona ao professor, realizar um acompanhamento mais detalhado de um aluno em relação aos demais integrantes de um mesmo grupo, de modo que, seja possível verificar o rendimento do aluno em comparação dos demais. Esse recurso tem como objetivo, possibilitar ao professor identificar o envolvimento do aluno com a atividade.

Ao acessar o sistema pela primeira vez o usuário é direcionado para a tela de autenticação. Realizado a autenticação no sistema, o professor tem acesso ao menu de opções, como mostra a Fig. 3.

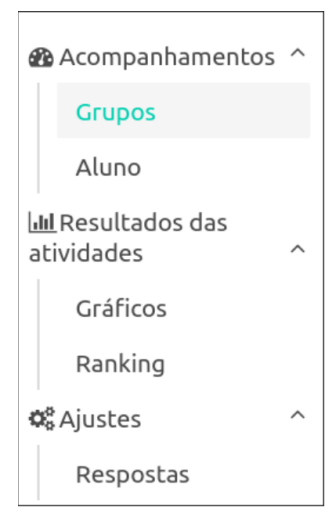

Figura 3: Menu de opções.

O menu é dividido em três categorias, sendo a primeira 
referindo-se aos acompanhamentos das atividades dos grupos ou dos alunos. A segunda mostra os resultados das atividades, tanto através de um gráfico, que compara o desempenho individual ou grupal, quanto através de um ranking de questões, que apresenta quais são as atividades com os menores índices de acertos.

A Fig. 4, mostra a tela de acompanhamento dos grupos.

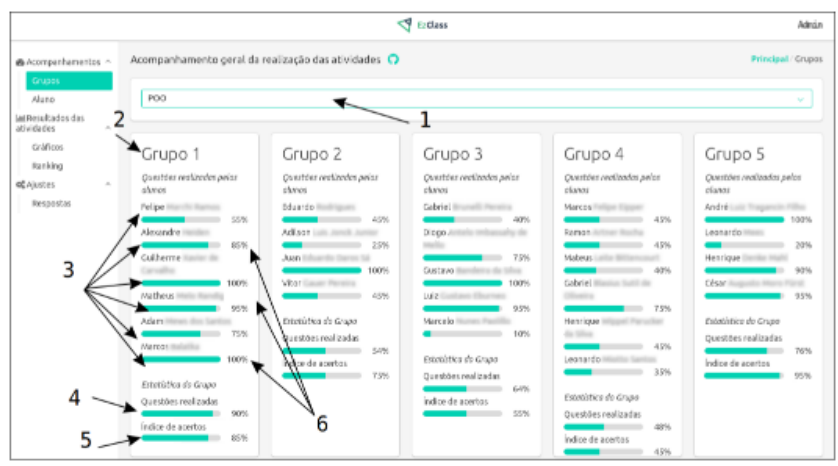

Figura 4: Acompanhamento geral dos grupos.

A tela acompanhamento de grupos representa os dados dos grupos conforme a atividade selecionada (item 1). Com isso, o professor tem a possibilidade de visualizar os blocos (item 2) correspondentes a cada grupo. Cada bloco é composto pelos participantes de um único grupo (item 3), um indicador de percentual de questões realizadas por integrante (item 3), um indicador de percentual de questões realizadas pelo grupo (item 4), e um indicador de percentual de acertos do grupo (item 5). 0 item 6 permite verificar se todos os alunos do grupo estão realizando a atividade, e os itens 4 e 5 correspondem à eficiência do grupo, o que possibilita ao professor tomar uma decisão imediata quanto ao desempenho do grupo. fico.

A Fig. 5 apresenta informações de um aluno em especí-

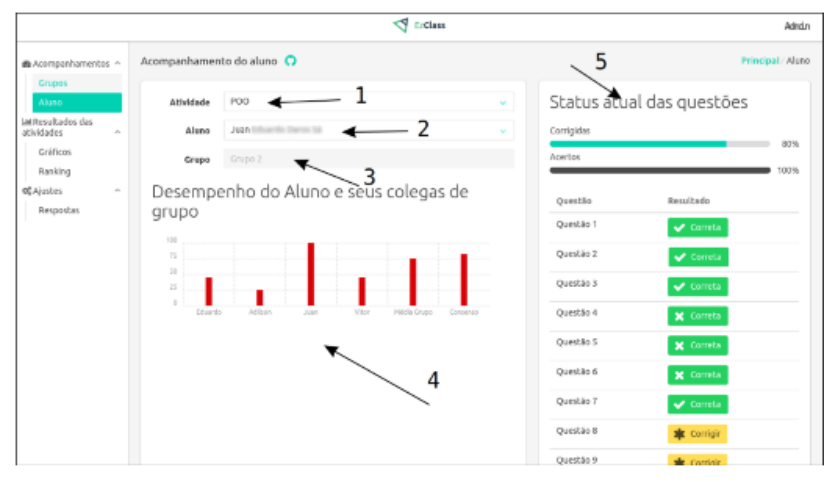

Figura 5: Acompanhamento do aluno.

Conforme destacado, o item 1 corresponde à seleção da atividade e o item 2 corresponde à seleção do aluno. O item 3 informa em qual grupo o aluno pertence, sendo esse, preenchido de forma automática. $\mathrm{O}$ item 4 representa um gráfico de desempenho em relação aos outros integrantes do grupo, por exemplo, o Juan possui desempenho melhor em relação aos outros membros do grupo. E o item 5 lista todas as questões da atividade com seus resultados, assim como questões que necessitam de correções manuais do tipo "abertas", bastando ao professor clicar no botão correspondente à questão, para realizar a correção.

A Fig. 6 apresenta todas as questões de uma atividade específica (item 1) em formato de tabela. A tabela (item 2) pode ser ordenada de acordo com as questões com maior número de acertos, isso permite que o professor visualize quais as questões que os alunos têm maior dificuldade. Essa informação pode ser utilizada como um indicativo para o professor da necessidade de revisar o conteúdo abordado pela questão.

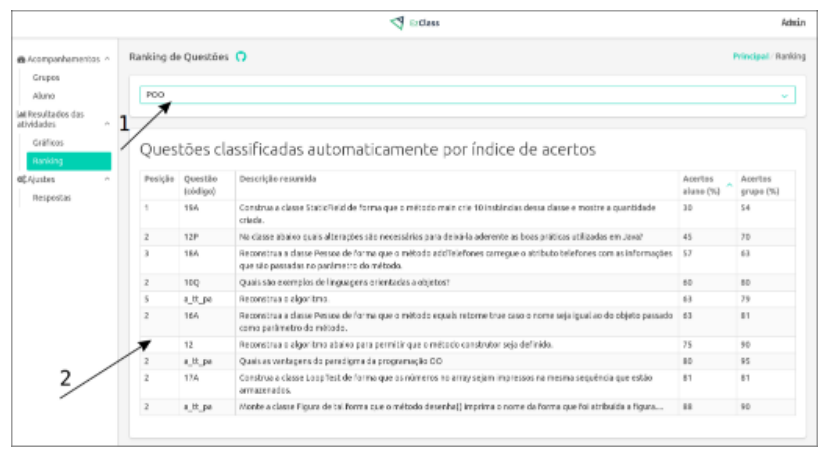

Figura 6: Classificação das questões.

A Fig. 7 mostra a tela de resultado geral da atividade. Nela são apresentados dois gráficos, os quais apresentam um resumo com os dados coletados da atividade selecionada no item 1 (destacado na imagem). O gráfico da esquerda corresponde ao resumo dos resultados individuais e o gráfico da direita corresponde ao resumo dos resultados dos grupos.

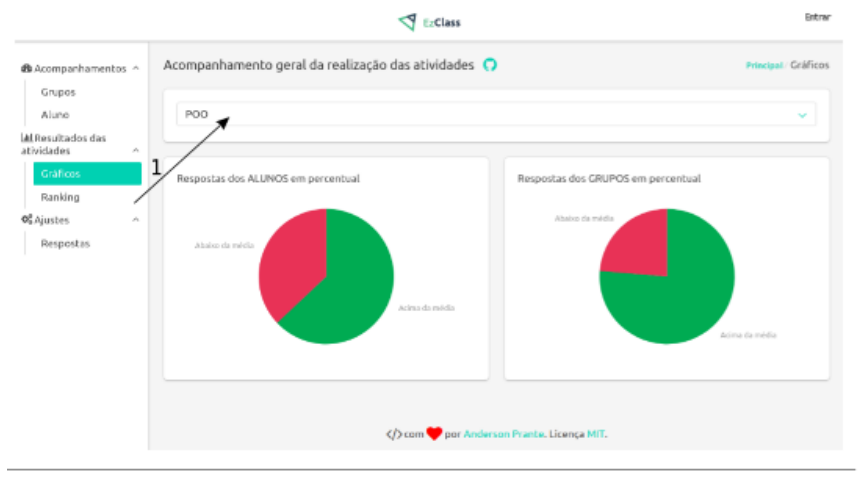

Figura 7: Resultado geral da atividade.

Adicionalmente foi desenvolvido uma tela do sistema que possibilita realizar ajustes nas questões cadastradas na base de dados do CLinClass como mostra a Fig. 8. Durante o cadastro das questões no CLinClass atualmente não 
existe uma forma de definir qual é a reposta correta para as questões do tipo múltipla escolha ou parson problem. Essa tela tem como característica apresentar uma lista completa com todas as questões cadastradas na base de dados, possibilitando, ao professor, inserir a informação referente à resposta correta para cada questão, no campo answer. Uma vez realizado essa configuração para as questões o sistema pode realizar as correções de forma automática.

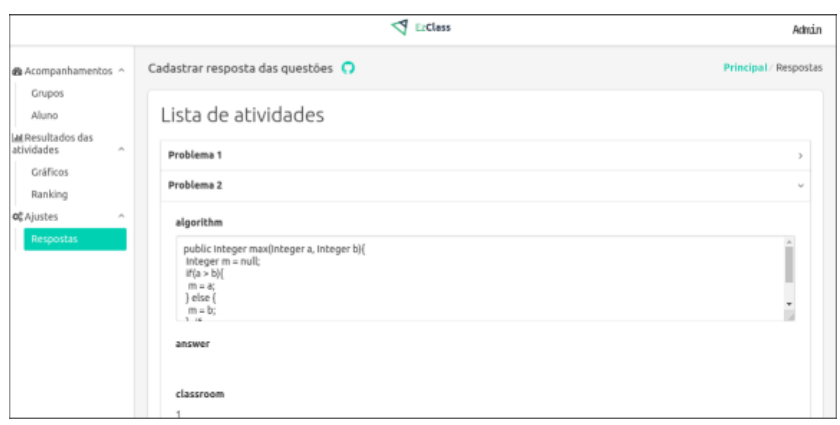

Figura 8: Tela de cadastro e ajuste de respostas para questões.

\section{Avaliação}

Nessa pesquisa foram realizadas duas etapas de avaliações. A primeira avaliação foi realizada por meio do método de inspeção semiótica, que teve como objetivo avaliar a comunicabilidade da interface do sistema. A segunda, foi a realização de um estudo de caso que teve como objetivo avaliar se a ferramenta pode ser utilizada como suporte didático para o professor. Nas subseções abaixo são apresentados os procedimentos das avaliações.

\subsection{Método de Inspeção Semiótica}

O Método de Inspeção Semiótica (MIS) pode ser aplicado por uma pessoa que não seja exclusivamente usuário do sistema, onde, por meio de um cenário previamente descrito, o avaliador possa se caracterizar como usuário do sistema. O cenário também é proposto com a finalidade de delimitar o escopo da avaliação. Desse modo, o cenário descrito para o avaliador do EZClass foi o seguinte: "Joana, professora do curso de Ciência da Computação, irá aplicar na sua nova turma uma atividade utilizando a ferramenta CLinClass, uma aplicação móvel colaborativa. Previamente, a professora realiza o cadastro das atividade que os alunos terão que responder, e define que a atividade deverá ser realizada em grupo. Ao chegar na sala de aula, passa instruções iniciais para a turma de como a ferramenta funciona e realiza o cadastro dos alunos. Com os cadastros realizados, é feita a formação dos grupos e, na sequência, é iniciada a atividade. Nessa etapa, a professora passa a monitorar e acompanhar o andamento das atividades na ferramenta." Segue abaixo a descrição da execução das etapas do MIS.

O método de inspeção semiótica foi realizado por dois avaliadores. A inspeção foi realizada em conjunto em uma seção de aproximadamente 40 minutos, nesse período, inicialmente foi descrito o cenário para os avaliadores, e a partir disto, os passos a seguir foram realizados. $O$ processo de avaliação foi realizado com a utilização de dados históricos registrados pelo CLinClass em outro período.

\section{- Análise dos signos metalinguísticos (etapa 1)}

Na primeira etapa foi avaliada a documentação referente à ferramenta. O EZClass até o momento não possui documentação oficial destinada para usuários, mas sim, um trabalho publicado sobre a versão de protótipo da ferramenta (Prante and Berkenbrock, 2018). Desta forma, os avaliadores identificaram a falta de documentação destinada aos usuários. Ainda nesta etapa, foi observada a inexistência da possibilidade da criação de novos usuários e recuperação de senhas. Não foram observados caixas de dicas nas telas do sistema. Contudo foram observados caixas de alerta durante a tentativa de acessar o sistema com usuários inválidos e o surgimento de mensagens de erros. Resultante desta etapa, observou-se que exitem poucos signos metalinguísticos.

\section{- Análise dos signos estáticos (etapa 2)}

Na tela inicial do sistema, observou-se os termos "login", "e-mail", "password" e "home", então, foi sugerido que estes termos fossem substituídos pelo idioma padrão do sistema, o português. Ao acessar a tela de grupos, a caixa de seleção apresentada estava em branco, indicando que era necessário selecionar uma turma para que os dados fossem apresentados. Após selecionar a turma, o sistema apresentou os grupos pertencentes respectivamente à turma selecionada, sendo possível visualizar quais eram os integrantes de cada grupo, qual o percentual de questões já realizadas por cada integrante, o percentual de questões realizadas por grupo e também o índice de acertos por grupo. O posicionamento dos elementos visuais foram considerados bons, com ressalva nas cores utilizadas na representação dos gráficos de barras que poderiam possuir cores variadas.

As cores dos gráficos gerados ao acessar o menu "Gráficos", foram consideradas boas, onde, a cor vermelha é utilizada para representar a parte correspondente aos alunos que ficaram com a nota abaixo da média e a cor verde é utilizada para representar os alunos que atingiram o resultado mínimo esperado segundo a atividade selecionada.

Ao acessar o menu "Ranking" localizado na categoria de "Resultados das atividades", foi necessário informar ao sistema qual a atividade que se desejava consultar. Após esse procedimento, o sistema apresentou uma tabela ordenada de acordo com os acertos individuais dos alunos. Desse modo, o avaliador teve a capacidade de visualizar quais eram as questões com maior índice de erros.

\section{- Análise dos signos dinâmicos (etapa 3)}

A análise dos signos dinâmicos foi realizada com dados históricos, desta forma poucos elementos dinâmicos puderam ser notados. A área do sistema correspondente ao acompanhamento de grupos (Fig. 4) tem como característica se modificar automaticamente enquanto há uma tarefa ainda em andamento. Contudo, na análise realizada, esse cenário não foi contemplado. Deste modo, as ações 
dinâmicas presenciadas pelos avaliadores corresponderam ao surgimento dos elementos de interface, ou seja, ações dinâmicas que ocorrem quando alguma ação fosse realizada. Nesse sentido, não houveram questionamentos.

\section{- Conclusão do Método de Inspeção Semiótica - MIS (etapa 4)}

O resultado geral sobre a análise dos signos metalinguísticos, estáticos e dinâmicos são focados na navegação do professor do ambiente do EZClass. No cenário utilizado, os problemas identificados concentram-se em partes pontuais do sistema. A primeira delas corresponde à terminologia, que não estava suficientemente adequada. A segunda observação, correspondeu à uniformidade no tom da cor verde utilizada em demasia no sistema, o que pode dificultar a percepção do professor no que se refere aos indicadores apresentados durante e após a execução das atividades pelos alunos/participantes.

No decorrer do processo do MIS uma metamensagem foi sendo modelada e refinada sempre que uma etapa era concluída. A metamensagem produzida a partir das análises dos signos foi a seguinte: "Você é um professor já habituado em utilizar sistemas computacionais. Você precisa acessar o sistema para poder acompanhar o andamento da atividade, de modo que seja possível identificar alunos com pouco engajamento na execução das tarefas. Esse sistema foi projetado para dar suporte a você (professor), que pode, por meio do sistema, acompanhar o andamento de atividades, verificar quais alunos estão com mais dificuldade, além de possibilitar identificar quais são as questões envolvidas na atividade que possuem um maior grau de dificuldade para os alunos."

Foi observado que o sistema não tem os recursos necessários para atender usuários que não possuem o hábito de utilizar sistemas computacionais, tal problema foi verificado pela ausência de documentação e menus de ajuda.

\section{- Avaliação qualitativa dos resultados do MIS (etapa 5)}

De modo geral, com base na metamensagem produzida a partir dos processos de análise, considera-se o resultado alcançado com a ferramenta satisfatório, uma vez que a metamensagem relata as principais características do sistema. Duas observações ditas pelos avaliadores que podem ser utilizadas em futuras versões do sistema: na tela de acompanhamento dos grupos, o primeiro bloco de informações, poderia corresponder à sumarização de todos os grupos presentes na atividade e na tela de gráficos (Fig. 8), os gráficos de pizza poderiam ser substituídos por gráficos de barra.

\subsection{Estudo de Caso}

O estudo foi realizado contou com cinco participantes, sendo eles professores atuantes da rede superior de ensino e possuindo em média 8,5 anos de experiência na docência, sendo o mínimo de 6 anos e o máximo de 16 anos.

O estudo de caso realizado teve como objetivo identificar e avaliar se os requisitos elicitados para o sistema EZClass permitem ao professor coordenar as atividades executadas no CLinClass. Para a realização do estudo de caso foi proposto e executado o protocolo descrito a seguir.
Inicialmente, realizou-se uma sessão de apresentação da ferramenta EZClass para todos os envolvidos no processo de avaliação, com tempo de duração de aproximadamente 20 minutos. Na sequência, cada um dos envolvidos fez uso do sistema individualmente com tempo de duração estimado em 15-20 minutos, o que possibilitou ao participante verificar as características presentes no sistema, investigar e validar as características técnicas relatadas no início da sessão. Ao final do tempo, foi solicitado ao participante que respondesse um total de doze questões, das quais, nove se referiam às características da plataforma, $\mathrm{e}$ as demais eram direcionadas ao participante da avaliação.

A Tabela 1 apresenta o resultado do estudo de caso aplicado aos avaliadores. Foram avaliados nove itens que permitiram analisar se a ferramenta dá o suporte necessário ao professor para atuar como coordenador nas atividades executadas por meio da ferramenta. Para validação, foram aceitos os itens que obtiveram um percentual igual ou superior à $60 \%$.

São duas as questões que ficaram com o índice de aceitação inferior ao esperado, são elas: a questão número um - a ferramenta permite visualizar dados de atividades em andamento, seu percentual baixo pode ser justificado de acordo com a forma que foi realizado o estudo de caso. A insatisfação corresponde ao fato de que o estudo de caso foi realizado com dados históricos (estáticos), dos quais não se tinha uma turma realizando uma atividade para que pudesse ocorrer ações dinâmicas na interface, por consequência disso, a visualização de atividades em andamento não foi avaliada; e a questão de número sete - A partir do diagnóstico, a metodologia de ensinoaprendizagem deveria ser modificada, seu baixo nível de aceitação pode estar relacionado com necessidade de que 0 professor deveria modificar a sua metodologia de ensinoaprendizagem em busca de melhores resultados, contudo, a questão número 5 corrobora com o fato de que é possível, com a ferramenta, diagnosticar problemas no processo de ensino-aprendizagem.

Os demais itens obtiveram um percentual igual ou superior à $60 \%$, fato que evidencia o potencial da ferramenta na coordenação de atividades realizadas em dispositivos eletrônicos. A ferramenta possibilita ao professor: acompanhar o envolvimento dos participantes nas atividades; saber quais são as questões que os alunos estão com mais dificuldades; identificar qual é o assunto que os alunos mais dominam naquele determinado espaço de tempo; perceber a evolução por meio da realização de trabalhos em grupo. E que, de fato, ter como métrica o tempo que o aluno passa tentando resolver uma atividade não é mais importante do que o esforço despendido por ele.

Foi questionado aos participantes, se já haviam utilizado outras plataformas de e-learning no papel de professor. E, caso positivo, quais foram essas plataformas. Todos afirmaram já ter utilizado o moodle, considerando que essa era a ferramenta adotada como padrão pela instituição, mas que atualmente utilizam uma plataforma adquirida em meados de 2014, que corresponde a um sistema integrado de gestão. Observou-se que, ao conversar com os participantes, eles relataram não ter tido contato com sistemas semelhantes ao EZClass em relação a forma de apresentação, organização e classificação das informações apresentadas. 
Tabela 1: Questionário aplicado aos participantes do estudo de caso

\begin{tabular}{|c|c|c|c|c|c|c|}
\hline $\begin{array}{l}\text { Questionário aplicado após experimento realizado } \\
\text { com dados histórico }\end{array}$ & DP & $\mathbf{D}$ & $\mathbf{C}$ & $\mathrm{CP}$ & $\begin{array}{l}\text { Soma das respostas }(\mathrm{C} \\
\text { e CP) }\end{array}$ & $\%$ respostas positivas \\
\hline $\begin{array}{l}\text { A ferramenta indica as questões com maiores índices } \\
\text { de erros }\end{array}$ & - & - & - & 5 & 5 & $100 \%$ \\
\hline $\begin{array}{l}\text { A ferramenta apresenta um diagnóstico do processo } \\
\text { de ensino-aprendizagem }\end{array}$ & - & 2 & 3 & - & 3 & $60 \%$ \\
\hline $\begin{array}{l}\text { A tela de resultados das atividades em forma de gráfi- } \\
\text { cos, tem como objetivo apresentar uma possível evo- } \\
\text { lução de uma atividade inicialmente realizada indivi- } \\
\text { dualmente e posteriormente realizada em grupo. Por } \\
\text { meio da observação dos gráficos pizzas apresentados } \\
\text { é possível ter essa percepção }\end{array}$ & - & - & 4 & 1 & 5 & $100 \%$ \\
\hline $\begin{array}{l}\text { Foi possível identificar uma melora no trabalho reali- } \\
\text { zado em grupo em relação ao individual }\end{array}$ & - & - & 3 & 2 & 5 & $100 \%$ \\
\hline $\begin{array}{l}\text { Uma das informações registradas pela ferramenta } \\
\text { CLinClass é o tempo em que o aluno leva para rea- } \\
\text { lizar uma atividade, essa informação não é relativa } \\
\text { em relação ao desenvolvimento pessoal do aluno }\end{array}$ & - & 1 & 3 & 1 & 4 & $80 \%$ \\
\hline
\end{tabular}

$\mathrm{DP}=$ Discordo plenamente $\mathrm{D}=$ Discordo $\mathrm{C}=$ Concordo $\mathrm{CP}=$ Concordo plenamente.

\section{Considerações Finais}

Em um processo de ensino e aprendizagem colaborativa, a intensa troca de informações entre os sujeitos envolvidos, apresentadas de forma estruturada, podem fornecer indicativos capazes de motivar a reflexão sobre as estratégias adotadas por discentes e sugerir novos mecanismos de interação.

O aplicativo CLinClass permite que os alunos realizem atividades de forma colaborativa, compartilhando conhecimento e realizando discussões em torno do conhecimento. Contudo, a ferramenta não fornece recursos para que o professor possa atuar como coordenador no ambiente. Os mecanismos de coordenação auxiliaram na elicitação de requisitos para o sistema EZClass com ênfase na coordenação. O sistema, proporciona, ao professor, realizar o acompanhamento das atividades, e que, influenciado pelas informações apresentadas pelo EZClass, possa ajustar sua prática pedagógica durante o processo de ensino.

Além de definir os requisitos para apoiar a coordenação, a apresentação do modelo de avaliação de comunicabilidade dos mecanismos na ferramenta EZClass, tem como objetivo garantir o sucesso da comunicabilidade da utilização pelo usuário final. Tal influência tende a crescer quando a ferramenta se destina a apoiar o trabalho ou aprendizagem colaborativa.

Os resultados obtidos com o estudo de caso indicam que o sistema é capaz de auxiliar um professor na realização do acompanhamento de atividades que os alunos estejam realizando por meio do uso do CLinClass, como também comprovou a eficácia das funcionalidades implementadas na ferramenta. Em comparação aos resultados obtidos com a realização do questionário durante o caso de uso, identificou-se que a ferramenta EZClass é capaz de realizar o monitoramento e acompanhamento individual das atividades de um aluno, e que tem como características exclusiva, a funcionalidade de monitorar atividades que são realizadas em grupo na ferramenta CLinClass.

Uma das limitações do trabalho está relacionada com o tamanho da amostra analisada no estudo de caso. Contudo, considerando que em um estudo de caso o resultado é dependente do contexto e muitas vezes só se consegue a generalização analítica estudando várias unidades de análise em contextos diferentes. Dessa forma, um dos direcionamento futuros do trabalho é estudar o impacto da ferramenta em diferentes contextos. Além disso, como trabalhos futuros sugere-se que a ferramenta contemple as seguintes funções:

- Uma característica do processo do método de inspeção semiótica é o de realizar uma verificação dos signos metalinguísticos da ferramenta e, no contexto desta pesquisa, constatou-se que o produto de software resultante não possui referencial teórico para usuários em geral. Desse modo, uma sugestão é a produção de documentação referencial para usuários, uma vez que essa demanda foi identificada mediante aplicação do MIS;

- Outra característica observada durante a etapa do MIS, diz respeito à inconsistência identificada no idioma empregado na interface do sistema. Essa característica já foi devidamente corrigida, contudo fica a possibilidade 
de implementar um modelo de internacionalização para que a aplicação passe a ter suporte a outros idiomas;

- Além disso, como forma de registrar uma sugestão apresentada por um dos avaliadores, na tela de acompanhamento dos grupos poderia ser apresentado um bloco com informações a respeito dos grupos, possibilitando outra forma de visualização do trabalho coletivo dos participantes.

\section{Referências}

Arnaut, B. M., Ferrari, D. B. and Oliveira e Souza, M. L. d. (2016). A requirements engineering and management process in concept phase of complex systems, 2016 IEEE International Symposium on Systems Engineering (ISSE), pp. 1-6. https://doi.org/10.1109/SysEng. 2016. 7753130.

Barbour, M. K., Grzebyk, T. and Eye, J. (2014). Any time, any place, any pace-really? examining mobile learning in a virtual school environment. https://doi.org/10. 17718/tojde. 4582.

Chorfi, A., Hedjazi, D., Aouag, S. and Boubiche, D. (2020). Problem-based collaborative learning groupware to improve computer programming skills, Behaviour and Information Technology pp. 1-20. https://doi.org/10. 1080/0144929X. 2020.1795263.

Correa, E. S. and Shinaigger, T. R. (2020). Smartphone como alicerce de metodologias ativas no ensino e aprendizagem da geografia, Educationis 8: 20-28. https: //doi.org/10.6008/CBPC2318-3047.2020.002.0003.

da Silva Marques, J. B., de Godoi, K. A. et al. (2021). Aplicativos de mensagens instantâneas em contextos de aprendizagem, Revista de Ensino, Educação e Ciências Humanas 22(1): 38-42. https://doi.org/10.17921/2447-8733. 2021v22n1p38-42.

dos Santos Fernandes, E. C. and Brasileiro, T. S. A. (2020). Aprendizagem móvel: O uso do smartphone como ferramenta pedagógica como recurso educacional, Revista AMAzônica 24: 382-401. https: //periodicos . ufam. edu . br/index.php/amazonica/article/view/7730/5424.

Duval, E. (2011). Attention please! learning analytics for visualization and recommendation, Proceedings of the 1st International Conference on Learning Analytics and Knowledge, LAK '11, Association for Computing Machinery, New York, NY, USA, p. 9-17. https : //doi.org/10.1145/ 2090116.2090118.

Ellis, C. A., Gibbs, S. J. and Rein, G. (1991). Groupware: Some issues and experiences, Commun. ACM 34(1): 39-58. https://doi.org/10.1145/99977.99987.

Felder, R. and Brent, R. (2007). Active and cooperative learning. Disponível em https: //www. engr.ncsu.edu/wp-content/uploads/drive/ 1PLmb19qPHpwYV7GFqUgWTHm5YNDzHV8V/2007-CLChapter. pdf.

Filvà, D. A., Guerrero, M. J. C. and Forment, M. A. (2014). Google analytics for time behavior measurement in moodle, 2014 9th Iberian Conference on Information Systems and Technologies (CISTI), pp. 1-6. https://doi .org/10. 1109/CISTI . 2014.6877095.

Fuks, H., Raposo, A. B., Gerosa, M. A. and Lucena, C. J. P. d. (2003). O modelo de colaboração $3 c$ e a engenharia de groupware, pp. 0-8.

Greenberg, S. (1989). The 1988 cscw: Trip report, SIGCHI Bull. 21(1): 49-55. https ://doi . org/10.1145/67880. 67886.

Grudin, J. (2001). News briefs, Computer 27(05): 19-21. https://doi.org/10.1109/MC.2001.10042.

Junior, C. B. d. S. and Oliveira, I. C. A. d. (2016). Learning analytics: Revisão da literatura e o estado da arte. Disponível em http://www.abed.org.br/ congresso2016/trabalhos/329.pdf.

Machado, L. D. P., Berkenbrock, C. D. M., Anselmo, G. and Siple, I. Z. (2018). Uma ferramenta colaborativa para apoiar a aprendizagem de programação de computadores, Revista Brasileira de Computação Aplicada 10(1): 2329. http://dx.doi.org/10.5335/rbcav10i1.7435.

Mazza, R. and Milani, C. (2004). Gismo: a graphical interactive student monitoring tool for course management systems, International Conference on Technology Enhanced Learning, Milan pp. 1-8.

Nascimento, K. A. S. d. and Filho, J. A. d. C. (2016). Uma revisão sistemática da literatura sobreaprendizagem móvel no ensino fundamental, REVISTA ELETRÔNICA PESQUISEDUCA 8(15): 121 - $136 . \quad$ Disponível em https://periodicos.unisantos.br/pesquiseduca/ article/view/510/pdf.

Pasini, J. F. S. (2020). Aplicativos e estratégias para desenvolver trabalho colaborativo e potencial dos alunos, Revista Pleiade pp. 05-16. http://dx.doi.org/10.32915/ pleiade.v14i31.

Pimentel, M., Gerosa, M. A., Filippo, D., Raposo, A., Fuks, H. and Lucena, C. J. P. d. (2006). Modelo 3c de colaboração para o desenvolvimento de sistemas colaborativos, pp. 58-67. Disponível em http://comissoes.sbc.org. $\mathrm{br} / \mathrm{ce}-\mathrm{sc} / \mathrm{sbsc} /$ anaisSBSC2006.pdf.

Prante, A. and Berkenbrock, C. D. M. (2018). O professor como coordenador em um ambiente móvel colaborativo de aprendizagem, Anais do IV COLBEDUCA - Colóquio Luso-Brasileiro de Educação, Vol. 3. Disponível em http: //www.revistas.udesc.br/index.php/ colbeduca/article/view/11383/8278.

Ramos, J., Cavalcanti, A., Santos, N. and Gomes, A. (2014). Mineração e visualização de dados educacionais: Identificação de fatores que afetam a motivação de alunos na educação a distância, Workshop de Educação e Informática Bahia Alagoas Sergipe.

Roschelle, J., Rafanan, K., Estrella, G., Nussbaum, M. and Claro, S. (2010). From handheld collaborative tool to effective classroom module: Embedding cscl in a broader design framework, Computers and Education 55(3): 1018 - 1026. https://doi.org/10.1016/j. compedu. 2010.04 . 012. 
Silva, J. C. S., Ramos, J., Rodrigues, R., Souza, F., Gomes, A. and Souza, H. (2016). Uma abordagem para integração do moodle com o framework shiny para learning analytics, Anais dos Workshops do Congresso Brasileiro de Informática na Educação 5: 930. http://dx.doi .org/10. 5753/cbie.wcbie.2016.930.

Stephens-Martinez, K., Hearst, M. A. and Fox, A. (2014). Monitoring moocs: Which information sources do instructors value?, Proceedings of the First ACM Conference on Learning@ Scale Conference, Association for Computing Machinery, New York, NY, USA, p. 79-88. https : //doi.org/10.1145/2556325.2566246.

Ting, Y.-L. (2013). Using mobile technologies to create interwoven learning interactions: An intuitive design and its evaluation, Computers and Education 60(1): 1 13. https://doi.org/10.1016/j. compedu. 2012.07.004.

Torres, P. L., Alcantara, P. R. and Irala, E. A. F. (2004). Grupos de consenso: uma proposta de aprendizagem colaborativa para o processo de ensino-aprendizagem, Revista Diálogo Educacional, Curitiba 4: 129-145. http: //dx.doi.org/10.7213/rde.v4i13.7052.

Varella, P. G., Vermelho, S. C., Hesketh, C. G. and Silva, A. C. C. d. (2002). Aprendizagem colaborativa em ambientes virtuais de aprendizagem: a experiência inédita da pucpr, Revista Diálogo Educacional, Curitiba 3: 11-27. http://dx.doi.org/10.7213/rde.v3i6.4804. 